\title{
Dust Production Rates in the Fomalhaut Debris Disk from SOFIA/FORCAST Mid-infrared Imaging
}

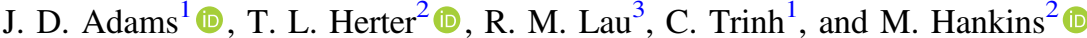 \\ ${ }^{1}$ Stratospheric Observatory for Infrared Astronomy, Universities Space Research Association, NASA/Armstrong Flight Research Center, \\ 2825 East Avenue P, Palmdale, CA 93550, USA \\ 2 Department of Astronomy, Cornell University, Space Sciences Building, Ithaca, NY 14853, USA \\ ${ }^{3}$ Division of Physics, Mathematics and Astronomy, California Institute of Technology, 1200 East California Boulevard, Pasadena, CA 91125, USA \\ Received 2018 March 14; revised 2018 June 23; accepted 2018 June 25; published 2018 August 2
}

\begin{abstract}
We present the first spatially resolved mid-infrared $(37.1 \mu \mathrm{m})$ image of the Fomalhaut debris disk. We use point spread function fitting and subtraction to distinctly measure the flux from the unresolved component and the debris disk. We measure an infrared excess in the point source of $0.9 \pm 0.2 \mathrm{Jy}$, consistent with emission from warm dust in an inner disk structure, and inconsistent with a stellar wind origin. We cannot confirm or rule out the presence of a pileup ring near the star. In the cold region, the $37 \mu \mathrm{m}$ imaging is sensitive to emission from small, blowout grains, which is an excellent probe of the dust production rate from planetesimal collisions. Under the assumptions that the dust grains are icy aggregates and the debris disk is in steady state, this result is consistent with the dust production rates predicted by Kenyon \& Bromley from theoretical models of icy planet formation. We find a dust luminosity of $(7.9 \pm 0.8) \times 10^{-4} L_{\odot}$ and a dust mass of 8-16 lunar masses, depending on grain porosity, with $\sim 1$ lunar mass in grains with radius $1 \mu \mathrm{m}-1 \mathrm{~mm}$. If the grains are icy and highly porous, meter-sized objects must be invoked to explain the far-infrared, submm, and $\mathrm{mm}$ emission. If the grains are composed of astronomical silicates, there is a dearth of blowout grains and the mass loss rate is well below the predicted dust production values.
\end{abstract}

Key words: circumstellar matter - infrared: planetary systems - planet-disk interactions

\section{Introduction}

Debris disks around stars are thought to be formed from a collisional cascade that occurs when planets form and stir planetesimals. Such collisions continually produce dust around the star. Smaller dust grains are blown out of the system by radiation pressure, while larger grains are pulled into the inner regions of the system by the Poynting-Robertson effect. However, the dust is replenished by the ongoing collisions that occur over the lifetime of the star. Kenyon \& Bromley (2008) have shown that the range of dust production varies with stellar mass, primordial disk mass, and stellar age. As protoplanets form around $1-3 M_{\odot}$ stars, dust production rises rapidly over 1-10 Myr, reaches a peak, then declines over the lifetime of the star. The peak dust production rates for $1-3 M_{\odot}$ ranges from $\sim 10^{19}$ to $\sim 10^{22} \mathrm{~g} \mathrm{yr}^{-1}$. More massive disks exhibit peak dust production earlier $(\sim 3 \mathrm{Myr})$ than less massive disks $(\sim 10 \mathrm{Myr})$. For $1-3 M_{\odot}$ stars with ages $>30 \mathrm{Myr}$, the range of predicted dust production rates narrows and becomes verifiable with observations. Specifically, the detection of small grains, coupled with calculations of their blowout timescales, can be used to determine the dust production rate in a debris disk that is in steady state.

Fomalhaut ( $\alpha$ PsA) is a nearby (7.7 pc; van Leeuwen 2007), young (440 Myr; Mamajek 2012) A3V star with an infrared (IR) excess and an active debris disk containing a cold submillimeter ring (Holland et al. 2003) that is interpreted as a Kuiper Belt analog. It also has a candidate planetary object, Fomalhaut b (Kalas et al. 2013). The disk was first discovered as IR excess in Infrared Astronomical Satellite (IRAS) observations (Aumann 1985). The IR excess is known to originate very close to the star, as seen in Spitzer/Infrared Spectrograph (IRS) spectra (Stapelfeldt et al. 2004) and nearinfrared (near-IR) interferometric imaging (Absil et al. 2009).
This excess has been interpreted as having a stellar wind origin (Acke et al. 2012) or as the signature of hot dust in the inner disk. Lebreton et al. (2013) proposed that the IR excess arises from a dust-producing belt at 1-2 au from the star, with an inner hot ring of dust located at $0.1-0.3 \mathrm{au}$. However, this model overpredicts the mid-infrared (mid-IR) flux (Su et al. 2016). Su et al. (2013) suggested that the dust arises from an asteroid belt, with the near-IR excess originating in hot, magnetically trapped nano grains near the star. The proposed asteroid belt lies near the ice line at $8-15$ au (Su et al. 2016), resulting in the production of dust, a drag-in disk from the Poynting-Robertson effect, and a pileup ring due to density enhancement near the silicate sublimation radius.

The cold belt is well defined (Acke et al. 2012; MacGregor et al. 2017) and exhibits apocenter glow (MacGregor et al. 2017) due to the eccentricity of the system. The disk has been observed in the mid-IR by Stapelfeldt et al. (2004), who used Spitzer/Multiband Imaging Photometer (MIPS) to obtain images of the disk at 24, 70, and $160 \mu \mathrm{m}$. At $24 \mu \mathrm{m}$, the ansai of the disk were detected; however, the Spitzer observations lacked the spatial resolution to completely resolve the disk from the star. Recently, Acke et al. (2012) used Herschel/Photoconductor Array Camera and Spectrometer (PACS; Poglitsch et al. 2010) and Herschel/Spectral and Photometric Imaging Receiver (SPIRE; Griffin et al. 2010) to image the disk in broad passbands at 70-500 $\mu \mathrm{m}$. Although the disk is resolved at $70 \mu \mathrm{m}$, the Herschel data are sensitive to large grains, and are less sensitive to the small grain population.

An estimate of the dust production rate requires knowledge of the dust grain composition. Fomalhaut shows an asymmetry in scattered light (Kalas et al. 2005), which could be interpreted as backscattering from large grains (Min et al. 2010). Fluffy aggregates have been considered to explain the observed scattering and thermal emission components to the dust spectral 
Table 1

Summary of SOFIA/FORCAST Observations of Fomalhaut under Plan ID 04_0064 and AOR ID 04_0064_1

\begin{tabular}{|c|c|c|c|c|c|}
\hline Mission ID & Start Time (UT) & End Time (UT) & Altitude (ft) & Starting Elevation Angle $\left(^{\circ}\right)$ & Calibration Uncertainty $(\%)$ \\
\hline 2016 Jul 12_FO_F319 & $09: 50: 35$ & 10:10:05 & 41,000 & 30 & 6 \\
\hline 2016 Jul 12_FO_F319 & 10:10:05 & $10: 54: 56$ & 42,000 & 33 & 6 \\
\hline 2016 Jul 12_FO_F319 & $10: 54: 56$ & $11: 44: 03$ & 41,000 & 39 & 6 \\
\hline 2016Jul 14_FO_F321 & $10: 49: 47$ & $11: 22: 55$ & 38,000 & 26 & 5 \\
\hline 2016 Jul 14_FO_F321 & $11: 22: 55$ & $11: 48: 33$ & 39,000 & 33 & 5 \\
\hline 2016 Jul 14_FO_F321 & $11: 48: 33$ & $12: 16: 41$ & 39,000 & 39 & 5 \\
\hline 2016 Jul 14_FO_F321 & $11: 48: 33$ & $12: 44: 16$ & 40,000 & 46 & 5 \\
\hline 2016 Jul 14_FO_F321 & $12: 44: 27$ & $13: 31: 10$ & 41,000 & 50 & 5 \\
\hline 2016 Jul 14_FO_F321 & $13: 31: 10$ & $13: 44: 20$ & 42,000 & 57 & 6 \\
\hline 2016 Jul 14_FO_F321 & $13: 58: 29$ & 14:10:08 & 42,000 & 59 & 6 \\
\hline 2016 Jul 18_FO_F323 & $10: 56: 20$ & $11: 29: 10$ & 40,000 & 25 & 5 \\
\hline 2016 Jul 18_FO_F323 & $11: 29: 10$ & $11: 59: 41$ & 40,000 & 31 & 5 \\
\hline 2016 Jul 18_FO_F323 & $11: 59: 54$ & $12: 25: 43$ & 41,000 & 38 & 5 \\
\hline 2016 Jul 18_FO_F323 & $12: 25: 43$ & $12: 51: 40$ & 42,000 & 43 & 5 \\
\hline 2016 Jul 18_FO_F323 & $12: 51: 40$ & $13: 15: 49$ & 42000 & 49 & 5 \\
\hline 2016 Jul 20_FO_F325 & 10:07:12 & $10: 46: 56$ & 39,000 & 34 & 5 \\
\hline 2016 Jul 20_FO_F325 & $10: 46: 56$ & $11: 26: 44$ & 39,000 & 41 & 5 \\
\hline 2016 Jul 20_FO_F325 & $11: 26: 55$ & $12: 04: 21$ & 39,000 & 48 & 5 \\
\hline 2016 Jul 20_FO_F325 & $12: 06: 35$ & $12: 39: 23$ & 39,000 & 52 & 5 \\
\hline 2016 Jul 20_FO_F325 & $12: 41: 21$ & $13: 16: 46$ & 41,000 & 55 & 5 \\
\hline
\end{tabular}

energy distribution (SED; Acke et al. 2012). These grains are similar to icy, porous cometary debris seen in the solar system (Fraundorf et al. 1982). Using fluffy aggregates, Acke et al. (2012) derived a mass loss rate of $2 \times 10^{21} \mathrm{~g} \mathrm{yr}^{-1}$ for $1-13 \mu \mathrm{m}$ grains in the cold component. However, this value is uncertain due to sparse data in the mid-IR.

An alternate interpretation of the Herschel data was present by Pawellek et al. (2014), who used Herschel data and astronomical silicate (Draine 2003) models to examine the grain size distribution in the spatially resolved component. No grains smaller than the blowout size were found, indicating that, if the grains are silicates, the smallest grain size formed in collisions is larger than the blowout size or the grains are rapidly blown out. These grains are iceless despite their location outside the ice sublimation zone.

Motivated to detect small grains and determine a precise measurement of the dust production rate, we obtained new midIR observations with the Stratospheric Observatory for Infrared Astronomy (SOFIA; Young et al. 2012). In Sections 2 and 3, we discuss SOFIA observations and results. In Section 4, we discuss dust models and results based on icy aggregate and astronomical silicate compositions. In Section 5, we derive the mass loss rates as a result of stellar radiation pressure. Section 6 contains a discussion of the results for both the point source and the Kuiper Belt analog. Our conclusions are presented in Section 7.

\section{Observations}

We observed Fomalhaut with the Faint Object InfraRed CAmera for the SOFIA Telescope (FORCAST) instrument (Herter et al. 2012) on SOFIA at a wavelength of $37.1 \mu \mathrm{m}$ (direct imaging mode), under SOFIA Program 04_0064. Observations were carried out over $\sim 10 \mathrm{hr}$ on four flights during Observing Campaign OC4-G (Southern Hemisphere deployment) in 2016 July (Table 1). FORCAST has a field of view of $3 ! 4 \times 3 ! 2$. The observations were performed with matched chopping and nodding (NMC mode) and dithering over a grid of nine positions spaced at $30^{\prime \prime}$. The off-source fields were chosen to be orthogonal to the apparent major axis of the disk in order to minimize the chop throw, thereby minimizing the amount of coma in the telescope beam. Typical dwell times in each nod beam were approximately $60 \mathrm{~s}$, including chopping inefficiencies. A small number of observations were identified to have been taken under aircraft door vignetting, which occurs when the telescope is near its elevation limit and causes significant background variations.

The raw data were processed using the pipeline described in Herter et al. (2013). This pipeline performs chop and nod subtraction and applies corrections for droop, detector nonlinearity, multiplexer crosstalk, and optical distortion. The chop, nod, and dither positions were aligned using the centroid of the star as a reference position. We averaged the aligned images (excluding the vignetted data), producing an image with an effective on-source exposure times of 24,302 s. The combined signals were individually weighted to account for the difference in signal between the matched beams and the non-matched beams. Finally, we subtracted residual background structure across the image caused by the presence of a bright central source (Lau et al. 2013) by using clipping and a second order polynomial fit to the background across each row. The rectified plate scale for the final image was 0 ".768 per pixel. The effective rms noise level was $\sim 0.8 \mathrm{mJy} \mathrm{pixel}^{-1}$.

Calibration factors at each level altitude were derived from the average photometric instrument response to standard stars observed during the observing campaign, with corrections for elevation angle and altitude. The calibration factors are for a flat-spectrum source. The calibration uncertainty was $\sim 6 \%$ (Table 1). We estimate a total uncertainty of $\sim 10 \%$ resulting from both calibration and flat-fielding errors.

\section{Observational Results}

\subsection{Imagery}

In Figure 1 (left panel), we show the processed $37 \mu \mathrm{m}$ image of Fomalhaut. The signal-to-noise ratio $(\mathrm{S} / \mathrm{N})$ per pixel is low (2) and therefore we have to rely on integrated signal for flux measurements. In Figure 1 (right panel), we show the processed $37.1 \mu \mathrm{m}$ image convolved with a Gaussian kernel to enhance 

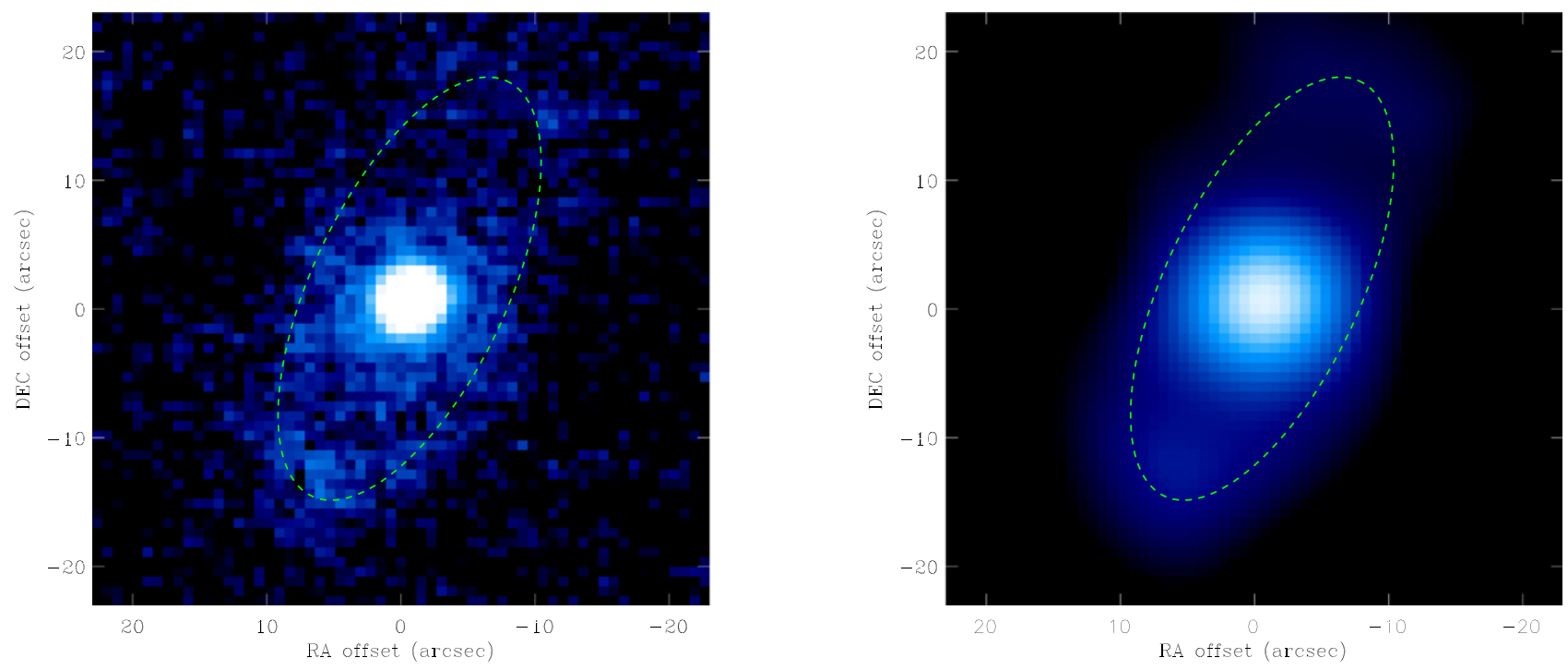

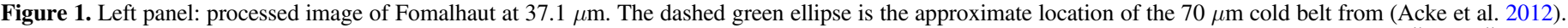
Right panel: smoothed image of Fomalhaut at $37.1 \mu \mathrm{m}$ with the same cold belt location. The smoothing kernel was a Gaussian with FWHM of 6 .' $2 \times 6$ ". 2 .

the $\mathrm{S} / \mathrm{N}$. The Gaussian kernel had a FWHM of 6 ". $2 \times 6$ "! 2 . The disk ansai are visible, with an $\mathrm{S} / \mathrm{N}$ of 48 per pixel in the $\mathrm{SE}$ ansa and an average $\mathrm{S} / \mathrm{N}$ of 25 in the NW ansa. For the subsequent analysis, we use unconvolved data for point spread function (PSF) subtraction and to derive the disk flux.

\subsection{PSF Subtraction}

In order to subtract the unresolved contribution from the disk flux, we performed PSF fitting on a standard star and the Fomalhaut point source. We constructed a model for the PSF consisting of a modified Airy function for diffraction, a polynomial component in the radial direction, and an elliptical Gaussian function for telescope jitter. The source of the a polynomial component is unclear, but it is prominent in the first Airy bright ring. A similar approach was used by $\mathrm{Su}$ et al. (2017), who fitted the FORCAST PSF at $35 \mu \mathrm{m}$ using a hybrid PSF based on a model and empirical, standard object PSFs. The model parameters were first adjusted to fit the radial profile of a standard star. We used observations of $\alpha$ Boo at $37.1 \mu \mathrm{m}$, taken during OC4-G, as a standard star reference for PSF fitting. The rms jitter values were 0 ". 84 and 0 " 55 in the cross-elevation and elevation directions, respectively. An Airy function for diffraction was modified by a third-order polynomial across the first bright ring. To enable us to subtract the PSF from the Fomalhaut image, we slightly adjusted to fit the radial profile of the Fomalhaut point source to account for variability in the PSF with telescope elevation. For Fomalhaut, the rms jitter values were $1 . " 1$ and 0 " 84 in the cross-elevation and elevation directions, respectively.

Figure 2 shows an image of $\alpha$ Boo, a radial profile of $\alpha$ Boo and the model fit to the data. Figure 2 also shows the image and residuals after the model has been subtracted from the data. The scatter in residuals is comparable to the background noise, indicating clean subtraction. Figure 3 shows analogous PSF fitting and subtraction for our Fomalhaut data. The PSFsubtracted image clearly shows the belt in the cold region.

\subsection{Flux Profile}

We generated a flux profile along the apparent major axis of the disk by summing the flux in the direction perpendicular to the apparent major axis. The width of the summed region was 23 !' 9 and the position angle of the apparent major axis was $156^{\circ}(\mathrm{E}$ of $\mathrm{N})$. Figure 4 shows this flux profile along the apparent major axis both with and without the PSF subtracted. The cold belt is spatially unresolved in the radial direction due to its narrow width (13 au; MacGregor et al. 2017). The outer edge of the SW ansa is located approximately $17^{\prime \prime}(131 \mathrm{au})$ and the outer edge of the NW ansa is located approximately 19 "! 3 (148 au) from the star (Figure 4). Taking into account blurring by the PSF, these results agree well with the semimajor axis of the outer edge of the belt as measured by the Atacama Large Millimeter/submillimeter Array (ALMA; $149 \pm 2$ au, MacGregor et al. 2017). The SE section shows emission from an inner disk. A fainter flux level in the NW section precluded detection of the inner disk. A faint halo is seen in the flux profile extending $\sim 10^{\prime \prime}$ beyond the edges of the ansai, but it is only marginally detected by FORCAST. In archival Herschel images $^{4}$ at $70 \mu \mathrm{m}$, this halo extends to a radius of $\sim 60^{\prime \prime}$ (460 au).

\subsection{Integrated Fluxes}

We used a tilted annulus to sum the flux over the region containing the Kuiper Belt analog and its halo. The annuli covered the $100-450$ au $\left(13^{\prime \prime}-58^{\prime \prime}\right)$ region to account for blurring by the PSF and the extent of the halo as seen in archival $70 \mu \mathrm{m}$ Herschel images. The inclination (65.6), position angle $\left(156^{\circ}\right)$, and eccentricity $(0.12)$ values were taken from Acke et al. (2012) and MacGregor et al. (2017). The average background per pixel was measured in a tilted annulus with radius $58^{\prime \prime}-60^{\prime \prime}$ and subtracted from each coadded pixel. We measured an integrated flux density of $2.0 \pm 0.2 \mathrm{Jy}$ in the cold region.

To compute the flux of the point source, we integrated the fitted model PSF. We applied a $15 \%$ color correction for the contribution from the photosphere. The photospheric flux density is expected to be $\sim 1.15$ Jy (Castelli \& Kurucz 2004). The color correction for unresolved warm dust is negligible. Our final result is $2.25 \pm 0.2 \mathrm{Jy}$ for a flat-spectrum source,

\footnotetext{
4 http://archives.esac.esa.int/hsa/whsa/
} 



Figure 2. PSF fitting and subtraction for $\alpha$ Boo. Upper left panel: FORCAST image of $\alpha$ Boo at $37.1 \mu \mathrm{m}$, averaged over the four OC4-G flights. Lower left panel: radial plot for $\alpha$ Boo (black dots), with flux densities in instrumental units. Model fits to the data (red points) include contributions from elliptical Gaussian jitter (green points) and a modified Airy function for diffraction and non-Gaussian components (blue points). Upper right panel: image of $\alpha$ Boo at $37.1 \mu \mathrm{m}$ after PSF subtraction. Lower right panel: radial plot of residuals after PSF subtraction.

which is color-corrected to $2.1 \pm 0.2 \mathrm{Jy}$ for the point source. This result yields an IR excess of $0.9 \pm 0.2 \mathrm{Jy}$ at $37.1 \mu \mathrm{m}$.

\section{Dust Modeling}

In order to characterize the dust populations in the debris disk, we performed dust modeling using a radiation field generated by the star and the assumption that the dust in the disk is in thermodynamic equilibrium. The luminosity $L_{g}$ of a single grain of radius $a$ is given by

$$
\begin{aligned}
L_{g} & =\pi a^{2} \frac{R_{*}^{2}}{r^{2}} \int F(\lambda) Q(\lambda, a) d \lambda \\
& =4 \pi^{2} a^{2} \int B(\lambda, T) Q(\lambda, a) d \lambda
\end{aligned}
$$

where $R_{*}$ is the stellar radius, $r$ is the distance from the star to the dust grain, $F(\lambda)$ is the stellar flux density at the surface of the star, $Q(\lambda, a)$ is the ratio of the absorption area to the geometric cross-sectional area, and $T$ is the equilibrium temperature of the dust grain.

\subsection{Radiation Field}

For the stellar flux density, we used a model stellar atmosphere with an effective temperature of $8750 \mathrm{~K}$ and solar metallicity from Castelli \& Kurucz (2004). We used an average distance from the star of 140 au for the cold region. The intervening material is optically thin based on its transparency in visible light images (Kalas et al. 2005).

\subsection{Grain Composition and Size Distributions}

For the grain composition, we adopted icy aggregate grains as suggested for Fomalhaut by Acke et al. (2012). We 

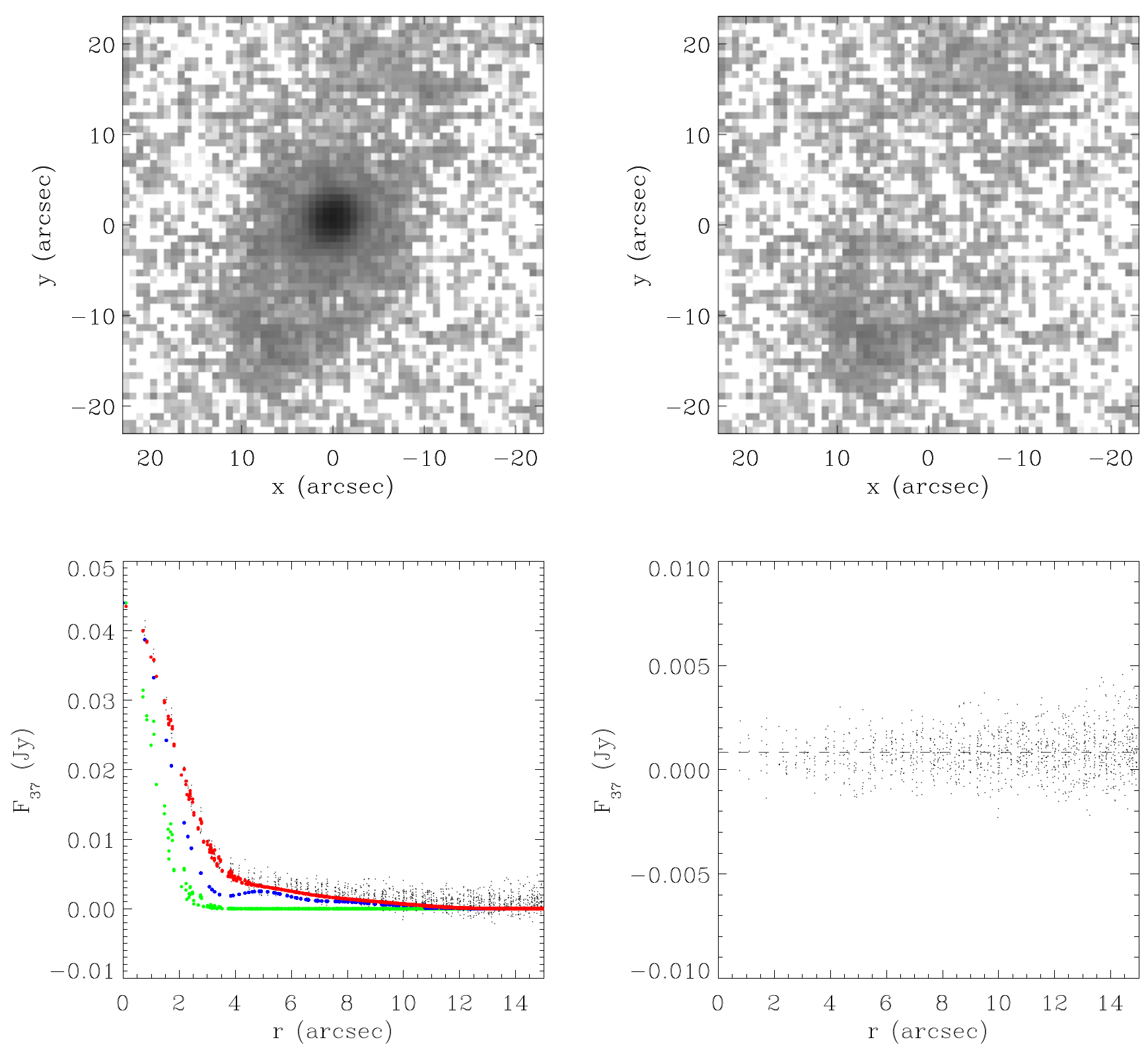

Figure 3. Analogous to Figure 2, but for Fomalhaut. The jitter model parameters were adjusted to fit the Fomalhaut data. The Fomalhaut PSF model uses the same modified Airy function as the $\alpha$ Boo PSF model.

considered a range of porosity, from compact grains to highly porous grains such as those seen in AU Mic (Graham et al. 2007). The icy grains are composed by mass of $22.5 \%$ silicates, $30.1 \%$ organics, and $47.4 \%$ water ice (Pollack et al. 1994; Kataoka et al. 2014), with a mean bulk density of $1.68 \mathrm{~g} \mathrm{~cm}^{-3} \cdot Q(\lambda, a)$ was computed analytically from the real and imaginary refractive indices and derivations given in Kataoka et al. (2014) for volume filling factors $f$ of 1.0, 0.5, 0.1, and 0.05 .

We also considered astronomical silicate grains as the source of dust emission (Draine 2003). They are compact, with a bulk density of $3.3 \mathrm{~g} \mathrm{~cm}^{-3}$ and a blowout radius of $\sim 5 \mu \mathrm{m}$ for Fomalhaut.

The grain size distribution $N(a)$ was modeled as $d N / d a \propto a^{q}$. The size distributions for blowout grains and for grains larger than the blowout size evolve differently, so we used a separate size distributions for the cases $a \leqslant a_{\text {blow }}$ and $a \geqslant a_{\text {blow }}$. The limits on the grain sizes are set by $a_{\min }$ and $a_{\max }$, the minimum and maximum grain sizes, respectively. For very large icy grains $(2 \pi a / \lambda \gg 1)$, the absorption coefficients approach $1-0.1 f$ and the scattering coefficients approach $1+0.1 f$ in the mid-IR and far-IR. $q, a_{\min }$, and $a_{\max }$ are the only free parameters in the dust model. The number of dust grains $N(a)$ and integrated luminosity of the dust populations are constrained by the observed SED.

\subsection{Modeling Results}

For icy grains in the cold region, we computed equilibrium temperatures that fall in the range 48-69 K. Figure 5 shows equilibrium temperature $T(a)$ as a function of grain radius $a$. The grains in the halo will be at a slightly lower temperature due to their larger distance from the star than the cold belt at a distance of $\sim 140 \mathrm{au}$, but their contribution to the integrated flux is small.

Figure 6 shows the SED for the cold region, including data from Spitzer/MIPS at $24 \mu \mathrm{m}$ (Stapelfeldt et al. 2004) and outer 


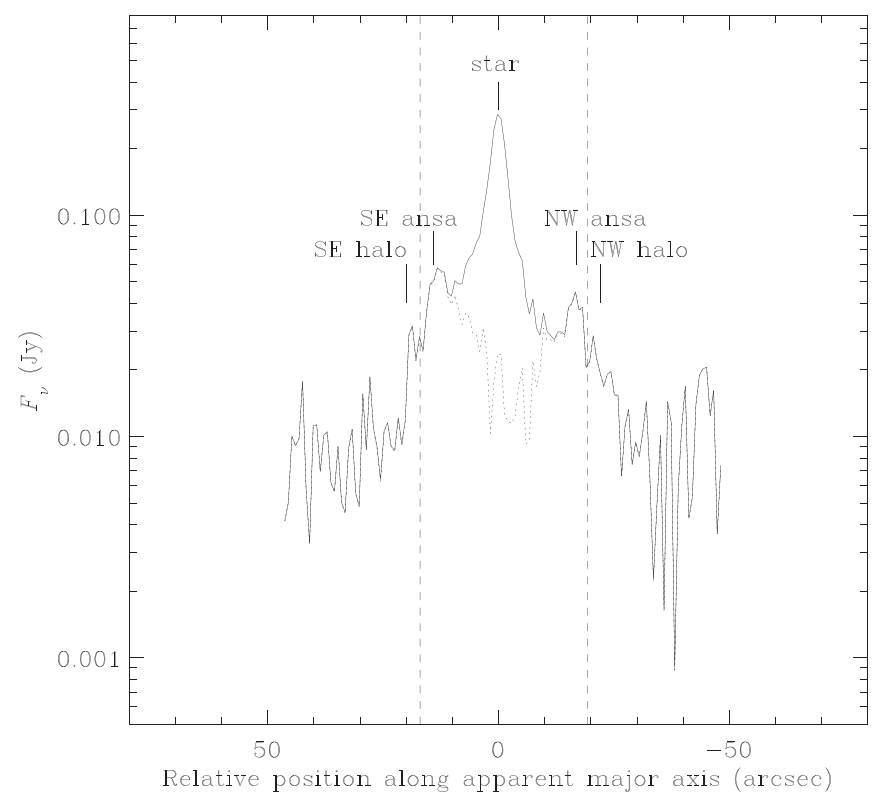

Figure 4. Integrated brightness profile along the apparent major axis, where the positive direction corresponds to the SE direction, and the negative direction corresponds to the NW direction. The width for the integration was 23!'9 (31 pixels). Profiles with (dotted line) and without (solid line) the PSF subtracted are shown. The edges of the ansai at $17^{\prime \prime}$ and -19 !. 3 are noted with dashed lines.

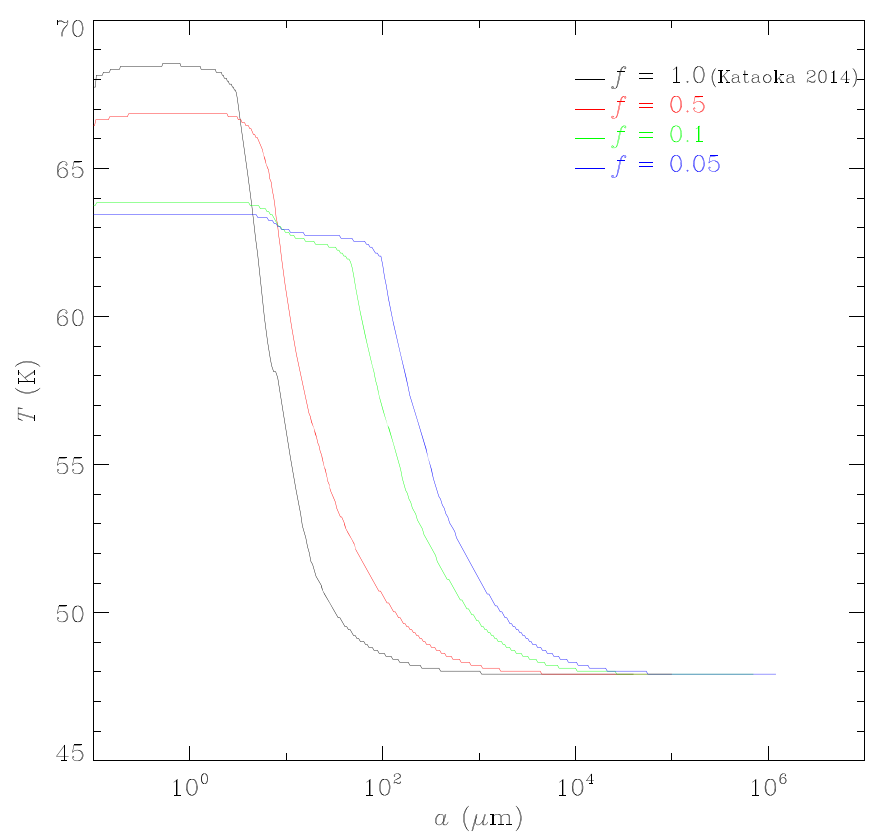

Figure 5. Grain equilibrium temperatures $T(a)$ vs. grain radius $a$ for icy grains with volume filling factors $f=1.0,0.5,0.1$, and 0.05 at a distance of $140 \mathrm{au}$.

disk fluxes at 70-500 $\mu \mathrm{m}$ from Acke et al. (2012). We note that the outer disk fluxes at 70-500 $\mu \mathrm{m}$ from Acke et al. (2012) were derived from a dust model that was convolved with the Herschel beams and fit to the Herschel images. Such an approach relies on grain composition and density distribution to separate the flux of the outer disk from that of the inner disk. Figure 6 also shows submillimeter data at $350 \mu \mathrm{m}$ (Marsh et al. 2005) and 450 and $850 \mu \mathrm{m}$ (Holland et al. 2003), as well as the integrated flux at $1.3 \mathrm{~mm}$ from ALMA (MacGregor et al. 2017).
Icy-grain model SEDs were fitted to the observed SED by eye $\left(\chi^{2} \approx 0.02\right)$ for volume filling factors $f$ of $1.0,0.5,0.1$, and 0.05 . Table 2 summarizes the best-fit model parameters and corresponding results for the cold belt. High-porosity grains require very large maximum grain sizes, up to $1.2 \mathrm{~m}$ for $f=0.05$. Figure 6 shows the SEDs for the model cases corresponding to the four values of volume filling factors. All of these cases can reproduce the observed SED. Consequently, the existing data set does not effectively constrain porosity. Integrating the model SEDs yields the dust luminosities, while integrating the size distribution and grain masses yields the dust mass. The dust mass values are listed in Table 2. The dust luminosity is tightly constrained at $(7.9 \pm 0.8) \times 10^{-4} L_{\odot}$, and the total dust mass ranges from $5.7 \times 10^{26} \mathrm{~g}$ for compact grains to $1.2 \times 10^{27} \mathrm{~g}$ for highly porous grains, or $\sim 8-16$ lunar masses. Uncertainties in the dust luminosity and dust mass were derived by varying the model parameters and examining the change in SED within flux uncertainties $(10 \%$ at most wavelengths).

We also fit the observed SED (Figure 7) with astrosilicate grains using the Debris Disk Simulator (Wolf \& Hillenbrand 2005) for two dust populations corresponding to the size ranges $a \leqslant a_{\text {blow }}$ and $a \geqslant a_{\text {blow. }}$. The results from this modeling are listed in (Table 2). The dust mass in blowout grains can be considered an upper limit because the SED can be fit with a single power-law size distribution with $a_{\text {min }}=a_{\text {blow }}$. The disk mass resulting from an astrosilicate model is $(6.6 \pm 0.7) \times$ $10^{25} \mathrm{~g}$, or about 0.9 lunar masses. Although the estimated dust mass from the astrosilicate grain model is lower than that for icy aggregate grains, the two compositions have similar masses for grains with $1 \mu \mathrm{m} \leqslant a \leqslant 1 \mathrm{~mm}$ (Table 2).

\section{Disk Modeling}

\subsection{Grain Orbits}

In order to compute the timescales for mass loss in the disk from radiation pressure on dust grains, we consider dust grains in orbit under the influence of gravity, radiation pressure, and centrifugal forces. Poynting-Robertson drag at the cold belt is negligible. We used the equations of motion in Harwit (1998), modified by the term for radiation pressure, to compute the orbits of blowout grains as conic sections from the cold belt (140 au) to the distant edge of the halo (450 au). The incident radiation that contributes to radiation pressure on a grain is $L_{\mathrm{RP}}=$ $R_{*}^{2} / r^{2} \int F(\lambda) \pi a^{2}\left(Q(\lambda, a)+\langle\cos \theta\rangle Q_{\text {sca }}(\lambda, a) d \lambda\right.$ (Tazaki \& Nomura 2015). The scattering efficiencies $Q_{\text {sca }}(\lambda, a)$ were calculated from the analytical formulae for the scattering of fluffy aggregates from Kataoka et al. (2014). Under the assumption of initial Keplerian motion, the time was integrated numerically to find the blowout radius and blowout time $\Delta t(a)$ (defined as the time for the grain to reach a distance 450 au from the star) for each value of $f$. For a hypothetical sample of small grains ( $a \ll a_{\text {blow }}$ ), our calculations agree with an analytical calculation for the blowout timescale from Lebreton et al. (2013), which is valid for cases where the radiation pressure dominates over the gravitational force and Poynting-Robertson drag. We note that our calculated value of $15 \mu \mathrm{m}$ for the blowout size for $25 \%$ porosity differs slightly from that of Acke et al. (2012; $13 \mu \mathrm{m})$. This difference may be due to their inclusion of iron sulfide in the grain composition. The presence of iron sulfide will increase the grain mass density and result in a smaller blowout size. For blowout grains, $\Delta t\left(a_{\text {blow }}\right) \lesssim 1100$ year, depending on 

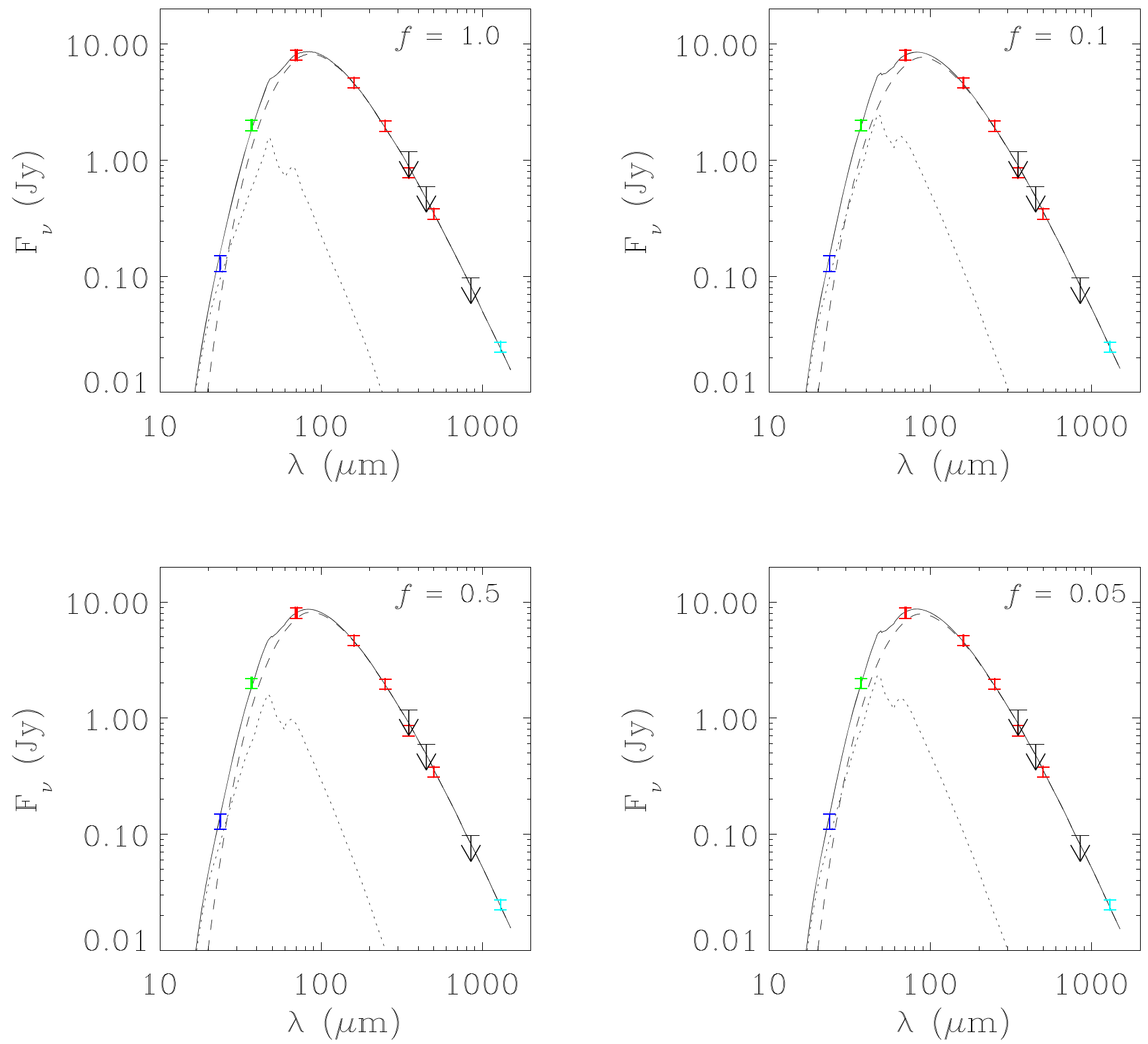

Figure 6. Observed SED and best-fitting models for the icy aggregate grain compositions. The SEDs for icy grains over a range of porosities $(f=1.0,0.5,0.1$, and 0.05; Kataoka et al. 2014) are shown. The contributions from blowout grains (dotted lines) and large grains (dashed lines) are shown, with the total SED depicted as solid black lines. Blue bar: Spitzer/MIPS flux from photosphere-subtracted image (Stapelfeldt et al. 2004). Green bar: FORCAST data (this work). Red bars: Herschel model for the outer disk from Acke et al. (2012). Cyan bar: ALMA data at $1.3 \mathrm{~mm}$ from MacGregor et al. (2017).The submillimeter data are at $350 \mu \mathrm{m}$ (Marsh et al. 2005), and 450 and $850 \mu \mathrm{m}$ (Holland et al. 2003). The submillimeter fluxes are shown as upper limits because they include contamination from the inner disk.

grain size. For our minimum grain size of $0.1 \mu \mathrm{m}, \Delta t \approx 230$ year for $f=1.0$ and $\Delta t \approx 700$ year for $f=0.05$.

\subsection{The Dust Production Rate}

The disk mass loss rate due to radiative blowout of small grains can be given as

$$
\dot{M}=\sum_{a=a_{\min }}^{a=a_{\text {blowout }}} N(a) m(a) / \Delta t(a)
$$

Although larger grains are produced in the collisional cascade, they are removed more slowly than the smaller grains, and consequently they contribute far less to the mass loss rate. Table 2 gives the value of $\dot{M}$ for each value of $f$. For fluffy aggregates, $\dot{M}$ can be expressed as $(1.2 \pm 0.5) \times 10^{21} \mathrm{~g} \mathrm{yr}^{-1}$, with uncertainties derived from uncertainties in porosity and observed fluxes.

For a disk containing an ongoing collisional cascade, we can compare the observed mass loss rate to a theoretical dust production rate. In order to make this comparison, we scaled the range of predicted dust production rates for $0.01-1.0 \mu \mathrm{m}$ grains from Kenyon \& Bromley (2008) to our range of grain sizes with $a_{\min } \leqslant a \leqslant a_{\text {blow }}$ based on the observed grain size distribution. The result yields the predicted dust mass that is produced every year for grain sizes in the range $a_{\min } \leqslant a \leqslant a_{\text {blow }}$. There is a range of predicted dust production rates to account for variations in initial disk mass. The range of dust production rates corresponding to the observed grain size 
Table 2

Summary of Dust Model Parameters and Results for the Cold Region

\begin{tabular}{|c|c|c|c|c|c|c|c|c|c|c|c|c|c|}
\hline \multirow[b]{2}{*}{ Composition } & \multirow[b]{2}{*}{$f$} & \multirow[b]{2}{*}{$\begin{array}{l}a_{\text {blow }} \\
(\mu \mathrm{m})\end{array}$} & \multicolumn{4}{|c|}{ Large Grains } & \multicolumn{6}{|c|}{ Blowout Grains } & \multirow[b]{2}{*}{$\begin{array}{l}M_{1 \mathrm{~mm}} \\
(\mathrm{~g})\end{array}$} \\
\hline & & & $q$ & $\begin{array}{l}a_{\min } \\
(\mu \mathrm{m})\end{array}$ & $\begin{array}{l}a_{\max } \\
(\mathrm{m})\end{array}$ & $\begin{array}{l}\log M_{d} \\
\text { (g) }\end{array}$ & $q$ & $\begin{array}{l}a_{\min } \\
(\mu \mathrm{m})\end{array}$ & $\begin{array}{l}a_{\max } \\
(\mu \mathrm{m})\end{array}$ & $\begin{array}{l}\log M_{d} \\
\quad(\mathrm{~g})\end{array}$ & $\underset{\left(\mathrm{g} \mathrm{yr}^{-1}\right)}{\log \dot{M}}$ & $\underset{\left(\mathrm{g} \mathrm{yr}^{-1}\right)}{\log \dot{M}_{\mathrm{P}}}$ & \\
\hline icy aggregates & 0.5 & 21 & -3.4 & 40 & 0.1 & $26.97_{-0.03}^{+0.04}$ & -3.5 & 0.1 & 40 & $23.78_{-0.20}^{+0.28}$ & $21.00_{-0.19}^{+0.03}$ & {$[20.66,21.12]$} & $25.83_{-0.03}^{+0.04}$ \\
\hline icy aggregates & 0.1 & 100 & -3.4 & 280 & 0.7 & $27.07_{-0.03}^{+0.02}$ & -3.5 & 0.1 & 280 & $24.02_{-0.11}^{+0.05}$ & $21.17_{-0.11}^{+0.04}$ & {$[20.68,21.14]$} & $25.82_{-0.03}^{+0.03}$ \\
\hline icy aggregates & 0.05 & 210 & -3.4 & 540 & 1.2 & $27.03_{-0.04}^{+0.02}$ & -3.5 & 0.1 & 540 & $23.99_{-0.18}^{+0.06}$ & $21.15_{-0.18}^{+0.07}$ & {$[20.69,21.15]$} & $25.84_{-0.04}^{+0.02}$ \\
\hline
\end{tabular}

Note. $M_{d}$ is the dust mass, $\dot{M}$ is the mass loss rate due to radiation pressure, $\dot{M}_{\mathrm{DP}}$ is the scaled dust production rate for grains with $a_{\text {min }} \leqslant a \leqslant a_{\text {blow }}$ from Kenyon \& Bromley (2008), and $M_{1 \mathrm{~mm}}$ is the mass in $1-1000 \mu \mathrm{m}$ (scaled with porosity) grains.

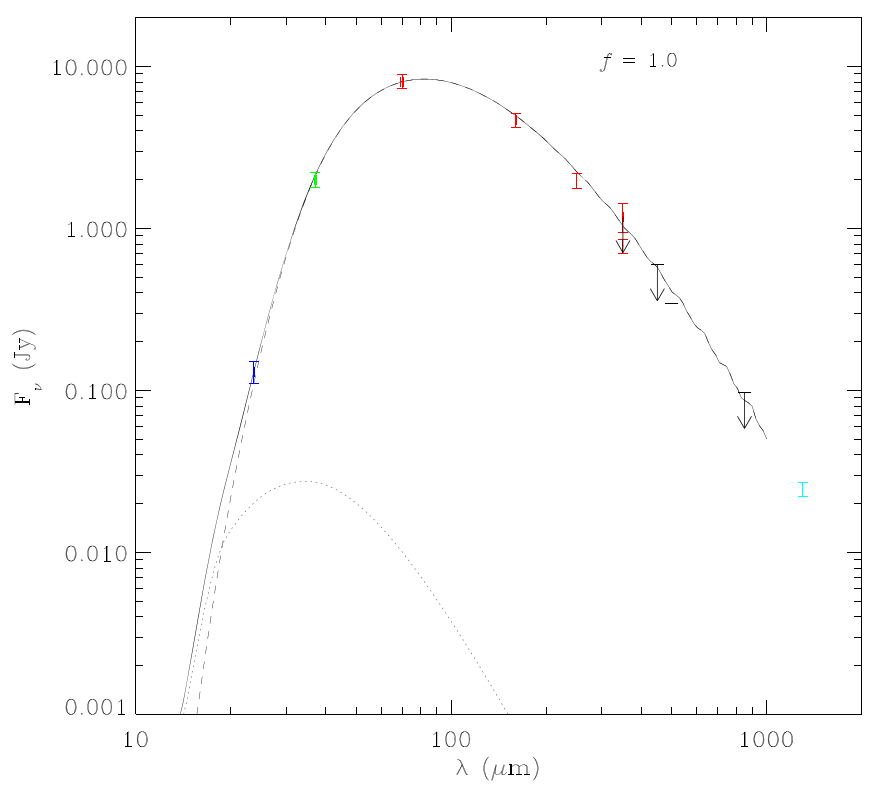

Figure 7. Observed SED and model fits for astrosilicates. Lines and data points are the same as those in Figure 6.

distribution for each value of $f$ is listed in Table 2. For icy grains, the predicted dust production rates agree with the observed mass loss rate. For astrosilicates, the mass loss rate falls below the predicted range of dust production rates.

We can also compare the observed disk mass to the predicted disk mass from a collisional cascade (Kenyon \& Bromley 2008). Table 2 lists the mass contained in compact grains with $1 \mu \mathrm{m} \leqslant a \leqslant 1 \mathrm{~mm}$ and the mass in the corresponding range for porous grains. The mass in these grains is $0.83-0.94$ lunar masses. This range is a factor of $\sim 2$ less than the predicted mass of $\sim 2$ lunar masses for a $1.7 M_{\odot}$ star (Kenyon \& Bromley 2008).

\section{Discussion}

\subsection{The Nature of the Central Source}

The nature of the inner region has been discussed by Absil et al. (2009), Acke et al. (2012), Lebreton et al. (2013), and Su et al. (2013, 2016). Acke et al. (2012) used near-IR (AKARI and Absil et al. 2009) and Herschel data to measure the slope of the IR excess from $\sim 1$ to $70 \mu \mathrm{m}$. They measured it as a power law in $F_{\nu}$ with index -0.8 . They proposed that this power-law index was consistent with a stellar wind origin for the IR excess



Figure 8. IR excess vs. wavelength for the point source at $24 \mu \mathrm{m}$ (blue point; Su et al. 2016), $37.1 \mu \mathrm{m}$ (green point; this work), and $70 \mu \mathrm{m}$ (red point; Acke et al. 2012; Su et al. 2013). The total emission from the inner disk model of Su et al. (2016) is shown both with (dotted-dashed line) and without (dotted line) the pileup ring. The expected IR excess from a stellar wind (dashed line; Acke et al. 2012) is shown.

(Panagia \& Felli 1975). If the IR excess originates in a stellar wind, the power law predicts an IR excess of $\sim 0.2-0.3 \mathrm{Jy}$ at $37 \mu \mathrm{m}$. Near-IR excesses and the Herschel $70 \mu \mathrm{m}$ excess flux from the central source source can be interpreted as originating from free-free emission associated with a stellar wind. However, ALMA measurements of the central source at $870 \mu \mathrm{m}$ (Su et al. 2016) indicate that the submillimeter flux is consistent with emission from a photosphere and not freefree emission. In Figure 8, we show the mid-IR SED of the central source with photometry from SOFIA/FORCAST (this work) and the predicted SED for free-free emission (Acke et al. 2012). The SOFIA/FORCAST photometry further rules out a stellar wind origin for the IR excess.

Lebreton et al. (2013) proposed that the IR excess in the inner region originates from warm dust near the star at 1-2 au. Such dust is within the ice sublimation radius. They predict an IR excess of $\sim 0.73 \mathrm{Jy}$ at $37 \mu \mathrm{m}$, which is slightly lower than the observed value, but still lies within the SOFIA/FORCAST uncertainties. However, these models overpredict the flux at 10-24 $\mathrm{m}$ (Su et al. 2016). 
$\mathrm{Su}$ et al. (2016) also proposed that the IR excess originates from warm dust, but they consider an asteroid belt at the ice line $(8-15 \mathrm{au})$. Astronomical silicate dust is produced in this belt, with larger grains dragged inward under PoyntingRobertson drag. The dragged-in grains form a warm disk and might be halted in a pileup ring at 0.23 au due to density enhancement near the silicate sublimation radius. The presence of a pileup ring can cause additional IR excess by $\sim 0.03 \mathrm{Jy}$ over the ice-line dust and warm disk. Figure 8 also shows the SED for warm dust from $\mathrm{Su}$ et al. $(2013,2016)$. The SOFIA/ FORCAST data support the warm disk hypothesis. However, the SOFIA data do not confirm or rule out the presence of a pileup ring, which results in additional excess of only $30 \mathrm{mJy}$ at $37.1 \mu \mathrm{m}$.

\subsection{Dust Production in the Kuiper Belt Analog}

Acke et al. (2012) derived a mass loss rate of $2 \times 10^{21} \mathrm{~g} \mathrm{yr}^{-1}$ using fluffy aggregate grains with $25 \%$ porosity under a $q=-3.5$ size distribution. For $25 \%$ porosity, we derive the slightly lower value of $\dot{M}=1 \times 10^{21} \mathrm{~g} \mathrm{yr}^{-1}$. We attribute the difference in size distributions to additional constraints on the observed SED from Spitzer/MIPS (Stapelfeldt et al. 2004), SOFIA (this work), and ALMA (MacGregor et al. 2017).

Highly porous (91\%-94\%) grains are observed in AU Mic (Graham et al. 2007) in scattered light. For Fomalhaut, highly porous grains would imply that the size distribution extends to meter-sized objects, if the material is composed of icy aggregates. Similar to mm-sized compact grains, such large objects are optically thick and highly (>99\%) absorbing of radiation. For highly porous grains, the blowout size is $\gtrsim 100 \mu \mathrm{m}$ and the minimum grain size is $\sim 60 \mu \mathrm{m}$ in the Fomalhaut Kuiper Belt analog. Such a size distribution is consistent with the proposed belt of micro-asteroids (Min et al. 2010).

If the grains are icy aggregates, the difference between the observed dust mass of $\sim 1$ lunar mass and the value of $\sim 2$ lunar masses predicted by Kenyon \& Bromley (2008) is not unexpected. The predicted dust mass corresponds to a different grain composition and porosity, with different absorption and scattering properties than the icy grains. Consequently, the predicted mass loss rate and accumulated disk mass will differ from the observed values for icy grains.

Icy aggregate dust grains can simultaneously reproduce the observed scattering and long wavelength thermal emission. If we extrapolate the predicted dust production rate for fine grains to our observed size distribution, we find it to be consistent with the theoretical predictions of icy planet formation Kenyon \& Bromley (2008). Therefore, the icy grain hypothesis is consistent with the observed emission and the expected dust production rates. However, we caution that the models of Kenyon \& Bromley (2008) differ from the Fomalhaut debris disk. The Fomalhaut Kuiper Belt analog is narrower $(13.5 \pm 1.8 \mathrm{au}$; MacGregor et al. 2017) than the theoretical disks, which span 30-150 au, so the theoretical disks have a larger volume of planetisimals that can be stirred by icy planets. In addition, the extrapolation of the dust production rates from fine $(0.01-1.0 \mu \mathrm{m})$ grains to larger grains $(a \geqslant 0.1 \mu \mathrm{m})$ relies on the assumption that dust is produced in steady state. An alternate interpretation could be that dust was produced 230-700 year ago and grains with $a<0.1 \mu \mathrm{m}$ have all been blown out of the system. In that case, the predicted dust production rate cannot be extrapolated because the dust production is instantaneous, rather than steady state. The steady-state assumption implies that the minimum grain sizes are larger than $\sim 1 \mu \mathrm{m}$, or in the case of highly porous grains, larger than $\sim 50 \mu \mathrm{m}$.

Both icy grain models and astrosilicate models can reproduce the observed SED. However, they differ in that the astrosilicate model indicates a dearth of blowout grains. This result confirms the results of Pawellek et al. (2014). If the grains are astrosilicates, they are produced at a lower rate or form with a minimum size that is larger than the blowout size.

\section{Conclusions}

We have used SOFIA/FORCAST to observe Fomalhaut at $37.1 \mu \mathrm{m}$. The observations have constrained the nature of the central source and the cold debris disk. The IR excess of the point source at $37.1 \mu \mathrm{m}$ is consistent with the presence of an asteroid belt at the ice line proposed by Su et al. (2013, 2016). The 37.1 excess in the point source rules out a stellar wind as the origin of IR excess in the central source.

Under the assumptions that the cold Fomalhaut debris disk is composed of icy aggregate dust grains and the disk is in a steady state collisional cascade, our observed mass loss rate is consistent with theoretical predictions for dust production rate from icy planet formation (Kenyon \& Bromley 2008). Icy grains remain a plausible dust grain type for the Fomalhaut because their presence around Fomalhaut would explain the observed scattered light, thermal emission, and the observed dust production rate. However, if the dust is composed of astrosilicates, the observed mass loss rate is less than the predicted range of dust production rates.

We thank K. Stapelfeldt for sharing his PSF-subtracted MIPS image of Fomalhaut and K. Su for sharing her inner-disk model SEDs. We thank the SOFIA ground crew, flight crew, and Mission Operations for their successful execution of the SOFIA observations. We also thank an anonymous referee whose suggestions led to the improvement of this manuscript. This work is based on observations made with the NASA/DLR SOFIA. SOFIA science mission operations are conducted jointly by the Universities Space Research Association, Inc. (USRA), under NASA contract NAS2-97001, and the Deutsches SOFIA Institut (DSI) under DLR contract 50 OK 0901.

Facilities: Spitzer, SOFIA, Herschel.

\section{ORCID iDs}

J. D. Adams (iD https://orcid.org/0000-0001-7645-8913

T. L. Herter (iD https://orcid.org/0000-0002-3856-8385

M. Hankins (iD https://orcid.org/0000-0001-9315-8437

\section{References}

Absil, O., Mennesson, B., Le Bouquin, J.-B., et al. 2009, ApJ, 704, 150

Acke, B., Min, M., Dominik, C., et al. 2012, A\&A, 540, 125

Aumann, H. H. 1985, PASP, 97, 885

Castelli, F., \& Kurucz, R. L. 2004, in IAU Symp. 210, Modelling of Stellar Atmospheres, ed. N. Piskunov et al. (Cambridge: Cambridge Univ. Press), poster A20

Draine, B. T. 2003, ARA\&A, 41, 241

Fraundorf, P., Walker, R. M., \& Brownlee, D. E. 1982, in IAU Coll. 61, Comet Discoveries, Statistics, and Observational Selection, ed. L. L. Wilkening (Tucson, AZ: Univ. Arizona Press), 383

Graham, J. R., Kalas, P. G., \& Matthews, B. C. 2007, ApJ, 654, 595

Griffin, M. J., Abergel, A., Abreu, A., et al. 2010, A\&A, 518L, 3 
Harwit, M. 1998, Astrophysical Concepts (2nd ed.; New York: Springer)

Herter, T. L., Adams, J. D., De Buizer, J. M., et al. 2012, ApJL, 749, L18

Herter, T. L., Vacca, W. D., Adams, J. D., et al. 2013, PASP, 125, 1393

Holland, W. S., Greaves, J. S., Dent, W. R. F., et al. 2003, ApJ, 582, 1141

Kalas, P., Graham, J. R., \& Clampin, M. 2005, Natur, 435, 1067

Kalas, P., Graham, J. R., Fitzgerald, M. P., \& Clampin, M. 2013, ApJ, 775, 56

Kataoka, A., Okuzumi, S., Tanaka, H., et al. 2014, A\&A, 568, 42

Kenyon, S. J., \& Bromley, B. C. 2008, ApJS, 179, 451

Lau, R. M., Herter, T. L., Morris, M. R., et al. 2013, ApJ, 775, 37

Lebreton, J., van Lieshout, R., Augereau, J.-C., et al. 2013, A\&A, 555, A146

MacGregor, M. A., Matrà, L., Kalas, P., et al. 2017, ApJ, 842, 8

Mamajek, E. E. 2012, ApJL, 754, L20

Marsh, K. A., Velusamy, T., Dowell, C. D., et al. 2005, ApJL, 620, L47
Min, M., Kama, M., Dominik, C., \& Waters, L. B. F. M. 2010, A\&A, 509, L6 Panagia, N., \& Felli, M. 1975, A\&A, 39, 1

Pawellek, N., Krivov, A., Marshall, J., et al. 2014, ApJ, 792, 65

Poglitsch, A., Waelkens, C., Geis, N., et al. 2010, A\&A, 518, L2

Pollack, J. B., Hollenback, D., Beckwith, S., et al. 1994, ApJ, 421, 615

Stapelfeldt, K. R., Holmes, E. K., Chen, C., et al. 2004, ApJS, 154, 458

Su, K. Y. L., De Buizer, J. M., Rieke, G. H., et al. 2017, AJ, 153, 226

Su, K. Y. L., Rieke, G. H., Defrére, D., et al. 2016, ApJ, 818, 45

Su, K. Y. L., Rieke, G. H., Malhotra, R., et al. 2013, ApJ, 763, 118

Tazaki, R., \& Nomura, N. 2015, ApJ, 799, 119

van Leeuwen, F. 2007, A\&A, 474, 653

Wolf, S., \& Hillenbrand, L. A. 2005, CoPhC, 171, 208

Young, E. T., Becklin, E. E., De Buizer, J. M., et al. 2012, ApJL, 749, L17 